\title{
Molecular Dynamic Simulation of Hydroxymethylglutaryl-CoA Reductase Inhibitors from Gnetum gnemon L. Seed Extract
}

\author{
Yuditya Artha, Arif Arrahman, Azminah, Arry Yanuar*
}

Yuditya Artha,

\section{Arif Arrahman, Azminah,} Arry Yanuar*

Faculty of Pharmacy, Universitas Indonesia, Depok 16424 West Java, INDONESIA.

Correspondence

\section{Arry Yanuar}

Faculty of Pharmacy, Universitas Indonesia, Depok 16424 West Java, INDONESIA.

Phone No: +62-21-7270031

E-mail: arry.yanuar@ui.ac.id

History

- Submission Date: 30-11-2017;

- Review completed: 23-04-2018;

- Accepted Date: 03-05-2018

DOI : 10.5530/pj.2018.4.134

Article Available online http://www.phcogj.com/v10/i4

\section{Copyright}

(c) 2018 Phcog.Net. This is an openaccess article distributed under the terms of the Creative Commons Attribution 4.0 International license.

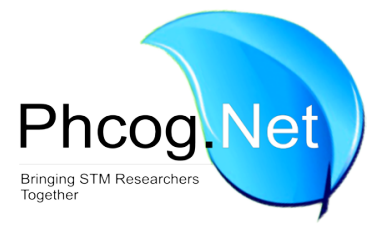

\begin{abstract}
Objective: Gnetum gnemon L. (melinjo) seed extract contained trans-resveratrol which has been shown to inhibit hydroxymethylglutaryl-CoA (HMG-CoA) reductase. Therefore it has a potent activity for lowering blood cholesterol. This study was carried out to determine the molecular dynamics simulation of HMG-CoA reductase inhibitors from Gnetum gnemon L. seed extract. Methods: Molecular dynamics simulation using AMBER was used. The simulation was set at $300 \mathrm{~K}$ as default temperature and $310 \mathrm{~K}$, average human body temperature. The main parameters of this study were ligand-residue interaction, binding affinity, root mean square deviation (RMSD), root mean square fluctuation (RMSF), hydrogen bonds analysis, molecular mechanics Poisson Boltzmann surface area (MMPBSA), and molecular mechanics generalized born surface area (MMGBSA). Results: In the simulation study, trans-resveratrol, trans-piceid, gnemonol $\mathrm{M}$, gnemonoside $\mathrm{B}$, viniferin and gnetin $\mathrm{C}$ had shown lower energy than HMG (PDB ID: MAH), the substrate of HMG-CoA Reductase. Free energy binding obtained from simulation was between 11.1 to $-31.38 \mathrm{kcal} / \mathrm{mol}$. Conclusion: The simulation at $310 \mathrm{~K}$ was preferable than $300 \mathrm{~K}$ as more interactions were performed and higher affinity was obtained.

Key words: Gnetum gnemon L, Hydroxymethylglutaryl-CoA reductase inhibitor, Molecular dynamics, Trans-resveratrol.
\end{abstract}

\section{INTRODUCTION}

Hypercholesterolemia is elevation the level of blood plasma cholesterol and one of the risk factor for cardiovascular diseases (CVD) occurrence (e.g., stroke and heart attack). Stroke places as the first leading cause of death in Indonesia according to data from World Health Organization in 2015. Food diet gives significant effect on CVD that caused by blood cholesterol level. ${ }^{1}$ Statin was invented in 1971 has a potential activity to inhibit cholesterol synthesis. Currently, statin and its analogs were chosen as primary prevention of CVD. ${ }^{2}$ From time to time, research on drugs, including statins was conducted to discover another alternative treatment of CVD. Development of drugs now focus on plants derived drugs to avoid unpredicted event during synthetic drugs research. ${ }^{3}$ Gnetum gnemon (melinjo) contains resveratrol abundantly including resveratrol derivatives: gnetin $\mathrm{C}$, gnemonoside $\mathrm{A}$ and gnemonoside D. ${ }^{4}$ Resveratrol inhibits HMG CoA to mevalonate conversion up to $32,4 \%$ while simvastatin reaches up to $43,0 \% .^{5}$ Another benefit of resveratrol is their intervention against dyslipidemia and obesity. ${ }^{6} \mathrm{An}$ experiment extracted melinjo seed to analyse several compounds composed within and its activity against hydroxymethylglutaryl-CoA reductase (HMGCR). ${ }^{7}$ The experiment shows dichloromethane extract gives highest inhibitory activity against HMGCR based on in vitro study. The extract predicted contains a com- pound that has potential inhibitory activity toward HMGCR and is expected performs higher affinity compared to HMG, the original substrate of HMGCR also the precursor of mevalonate.

Molecular dynamics simulation provides the structure as a dynamic system with transformed continuously structural conformation. ${ }^{8}$ The computational study, molecular dynamics (MD) were carried as a complementary study to in vitro experiment. The affinity each of the extracted compound is compared to statins and HMG by employing the computational method.

\section{MATERIALS AND METHODS}

Viniferin, piceid, gnemonol $\mathrm{M}$, gnetol, isorhapontigenin, latifolol, resveratrol, gnemoside $\mathrm{B}$, gnetin $\mathrm{C}$, HMG, rosuvastatin, and pravastatin were selected for the ligands in this study. These ligands were parameterized by Sander adding AM1-BCC charge in Antechamber. ${ }^{9}$

Best energy conformation from docking study was chosen to be used as preparation material on MD. Several studies state their inhibitory activity towards HMGCR. ${ }^{4-6,10 .}$ Thus, Piceid and Resveratrol were included in the simulation as well to rescore binding affinity. The interaction between residues and ligands was visualized by LigPlot. ${ }^{11}$

Cite this article: Artha Y, Arrahman A, Azminah, Yanuar A. Molecular Dynamic Simulation of Hydroxymethylglutaryl-CoA Reductase Inhibitors from Gnetum gnemon L. Seed Extract. Pharma$\operatorname{cog}$ J. 2018;10(4):793-7. 
The simulation was running in Amber using pmemd.cuda in GPU environment. ${ }^{12}$ ADP as the cofactor was included in this simulation and prepared. The required files were downloaded at http://research.bmh. manchester.ac.uk/bryce/amber. ${ }^{13}$ Simulation was set at $300 \mathrm{~K}$ and $310 \mathrm{~K}$, water TIP3P octahedron was selected as the solvate with $12.0 \AA$ size. The charges of each ligand were generated using antechamber. $\mathrm{Na}^{+}$as counter ion was added to create a system with neutralized $\mathrm{pH}$. Dynamics of the system was observed and analyzed for 20 ns subsequently visualized by VMD. ${ }^{14}$ Minimisation was carried to equilibrate the solvated complex (volume pressure npts nvts constant). Constant heat and density until 600 ps were preceded by 50 ps heat and density equilibration, time step was set at two femtoseconds. The residues from $1-786$ were monitored during the equilibration. Hydrogen bond interactions were limited to less than $3 \AA$ and $60^{\circ}$ for the distance and bonding angle respectively.

\section{RESULTS AND DISCUSSIONS}

\section{Molecular dynamic}

PDB ID 1HW8 was selected as macromolecule target and was validated using redocking Mevastatin to the macromolecule using Autodock. ${ }^{15,16}$

\section{Equilibration}

The system was equilibrated in $300 \mathrm{~K}$ and $310 \mathrm{~K}$ until it reached temperature stability at $300 \mathrm{~K}$ and $310 \mathrm{~K}$ precisely, density close to water $(1 \mathrm{~g} / \mathrm{ml})$

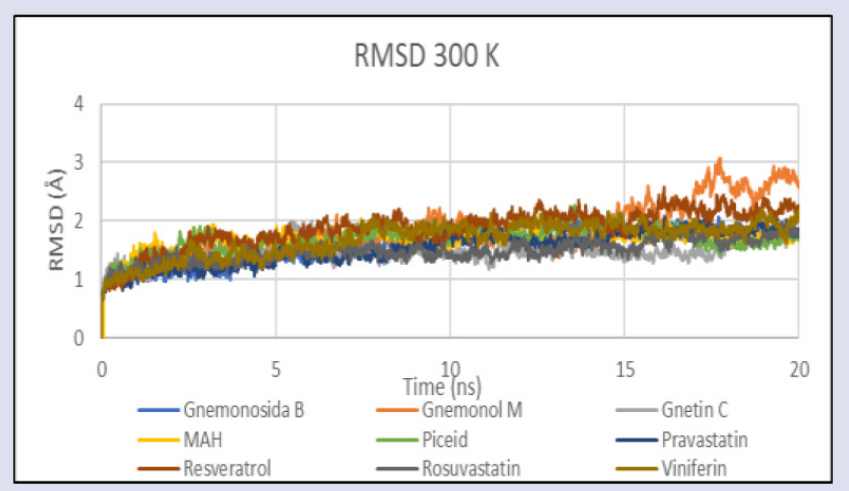

Figure 1: RMSD plot of residues on Microsoft Excel at $300 \mathrm{~K}$ during 20 ns.

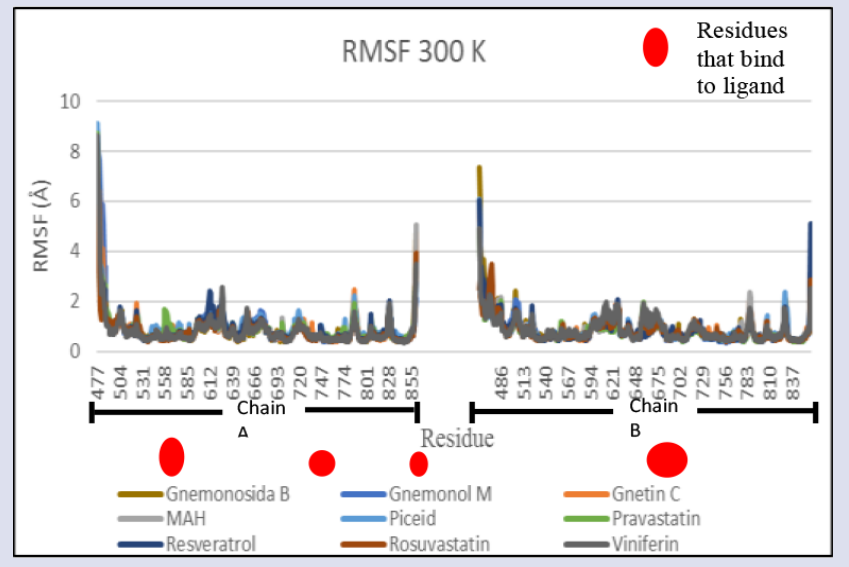

Figure 3: RMSF plot of residues on Microsoft Excel at $300 \mathrm{~K}$ during 20 ns. at atomic pressure (1 atm). The stability of the system was examined by observing their plot of density, total energy, and temperature visually.

\section{RMSD AND RMSF}

RMSD and RMSF measures system dynamic in which higher values signify poor stability. A system with $310 \mathrm{~K}$ tends to be more fluctuate than $300 \mathrm{~K}$ as shown on RMSD (root mean square deviation) graph Figure 1 and $2 . \mathrm{RMSD}=0.00$ indicate perfect overlapping between structure coordinate and reference coordinate, lower value signifies more likeness towards reference coordinate. RMSF (root mean square fluctuation) is a measurement of average primary chain mobility. ${ }^{17}$ In contrast, a variation of temperature did not show the meaningful impact on RMSF Figure 3 and 4 .

\section{Hydrogen bond}

A hydrogen bond is an interaction between $\mathrm{X}-\mathrm{H}------\mathrm{A}$, wherein $\mathrm{H}$ is positively charged, and A is partially or entirely negatively charged. ${ }^{18}$ A hydrogen bond is classified based on the occupancy percentage: weak (25-50\%), strong (50-75\%) and very strong (75-100\%). ${ }^{19}$ System $310 \mathrm{~K}$ formed more hydrogen bonding and obtained higher occupancy implying higher temperature induces the system to be more active, consequently established more opportunity to initiate contacts with neighbouring atoms (Table 1, 2).

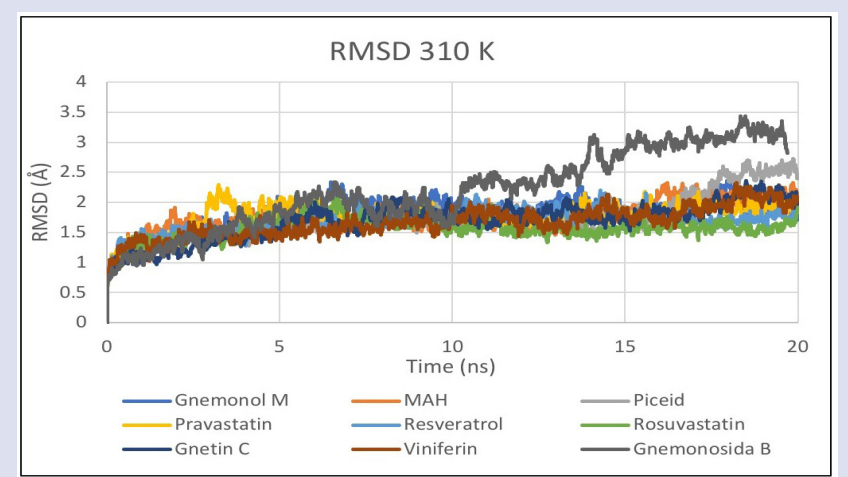

Figure 2: RMSD plot of residues on Microsoft Excel at $310 \mathrm{~K}$ during 20 ns.

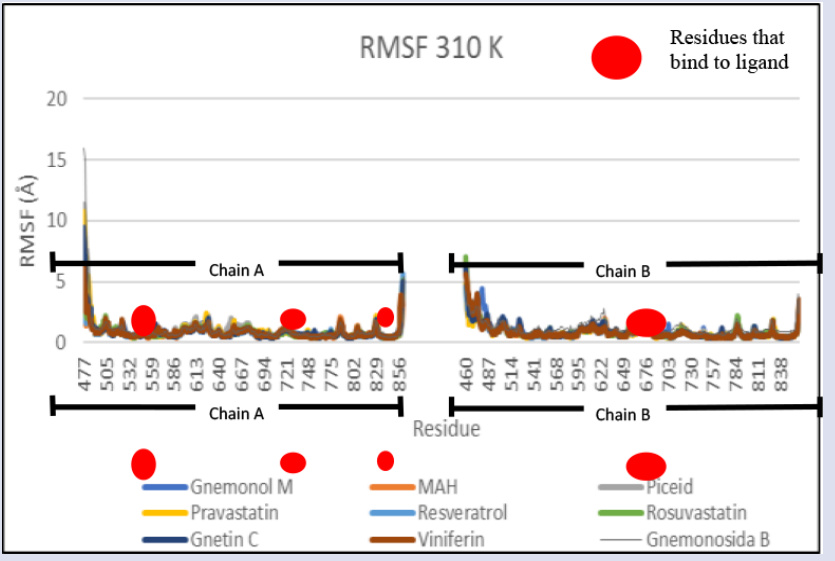

Figure 4: RMSF plot of residues on Microsoft Excel at $310 \mathrm{~K}$ during 20 ns. 


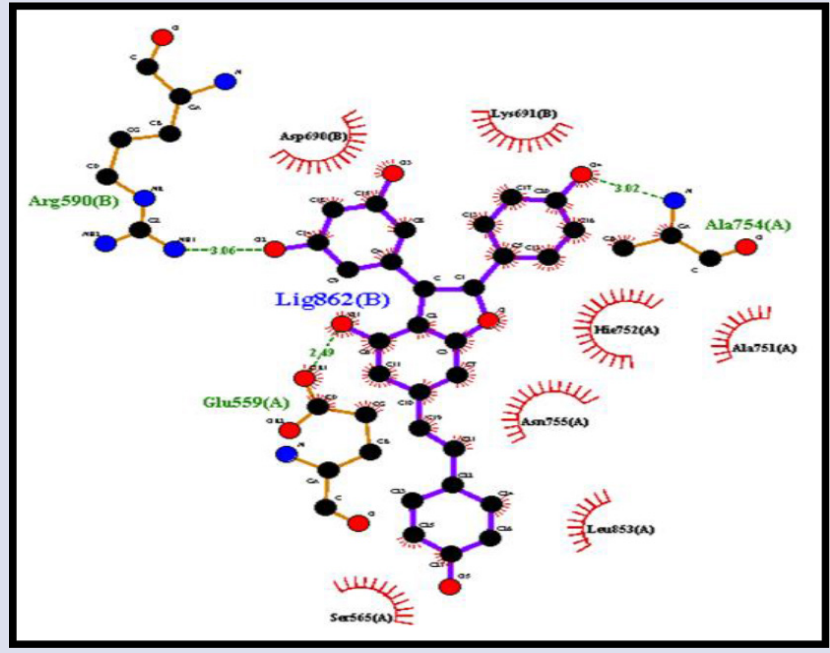

Figure 5: Interaction of Gnetin C during $20 \mathrm{~ns}$ simulation at $310 \mathrm{~K}$.

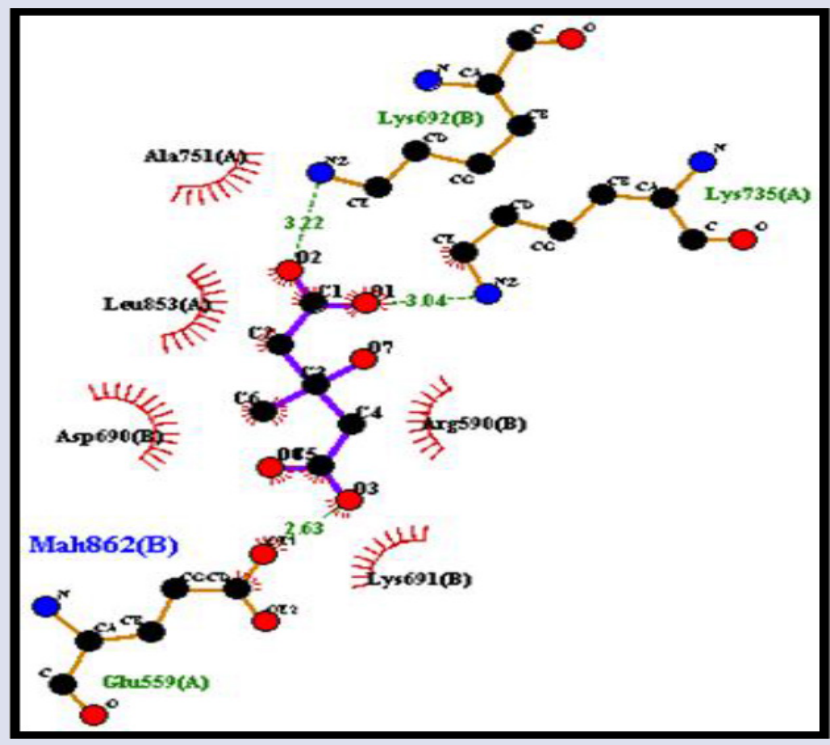

Figure 6: Interaction of HMG during 20 ns simulation at 310.

Gnetin C performed the highest number of total hydrogen bonds at $310 \mathrm{~K}$, 5.361 bonds. Compared to the system at $300 \mathrm{~K}$, gnetin $\mathrm{C}$ produced 2,397. The difference in total hydrogen bonding resulted in a significant gap of energy binding affinity between $300 \mathrm{~K}$ and $310 \mathrm{~K}$ during gnetin $\mathrm{C}$ simulation. Hence hydrogen bonds were a critical parameter to be highly considered on each ligand (Table 3, 4).

Although hydrogen bonding was essential in this study, hydrophobic interactions play a significant role as well. HMG as the substrate has less hydrophobic groups compared to other ligands resulting lower affinity as shown in Table 4.

Active binding sites 1HW8: Ser ${ }^{684}$, Asp ${ }^{690}$, Lys ${ }^{691}$, Lys ${ }^{692}$, Lys $^{691}$, Lys $^{735}$ which essential for hydrogen bonding and $\mathrm{Leu}^{562} \mathrm{Val}^{683}, \mathrm{Leu}^{853}, \mathrm{Ala}^{856}$, $\mathrm{Leu}^{857}$ important residues for hydrophobic interaction. ${ }^{20}$ In this study, ligands performed less contact with neighbouring hydrophobic amino acids compared to the statin.
Table 1: Total amount of hydrogen bonds during $20 \mathrm{~ns}$.

\begin{tabular}{ccc}
\hline Ligand & \multicolumn{2}{c}{ Total Hydrogen Bonds } \\
\cline { 2 - 3 } & $300 \mathrm{~K}$ & $310 \mathrm{~K}$ \\
\hline Gnemonol M & 1,845 & 2,195 \\
Gnetin C & 2,397 & 5,361 \\
HMG & 2,088 & 2,731 \\
Piceid & 8,857 & 6,972 \\
Pravastatin & 5,771 & 7,001 \\
Resveratrol & 2,054 & 1,992 \\
Rosuvastatin & 7,917 & 8,856 \\
Viniferin & 4,073 & 4,380 \\
Gnemonoside B & 3,265 & 1,733 \\
\hline
\end{tabular}

Table 2: Hydrogen bonds occupancy at $300 \mathrm{~K}$ during $20 \mathrm{~ns}$.

\begin{tabular}{|c|c|c|c|c|}
\hline No & Ligand & Donor & Acceptor & $\begin{array}{c}\text { Occupancy } \\
\text { (\%) }\end{array}$ \\
\hline 1 & Gnemonol M & LIG788-Side-O3 & HIE861-Side-OXT & $64.95 \%$ \\
\hline \multirow[t]{2}{*}{2} & Gnemonoside & UNK788-Side-O4 & GLU665-Side-OE1 & $50.25 \%$ \\
\hline & & UNK788-Side-O4 & GLU665-Side-OE2 & $32.85 \%$ \\
\hline 3 & Gnetin C & LIG788-Side-O4 & ALA751-Main-O & $28.5 \%$ \\
\hline \multirow[t]{2}{*}{4} & HMG & LYS735-Side-NZ & MAH788-Side-O1 & $38.75 \%$ \\
\hline & & LYS735-Side-NZ & MAH788-Side-O2 & $37.20 \%$ \\
\hline \multirow[t]{6}{*}{5} & Piceid & LIG788-Side-O1 & GLU559-Side-OE1 & $54 \%$ \\
\hline & & LIG788-Side-O1 & GLU559-Side-OE2 & $52.5 \%$ \\
\hline & & LIG788-Side-O6 & ASP690-Side-OD1 & $48.3 \%$ \\
\hline & & LIG788-Side-O6 & ASP690-Side-OD2 & $45.1 \%$ \\
\hline & & LIG788-Side-O2 & GLU559-Side-OE1 & $42.45 \%$ \\
\hline & & LIG788-Side-O7 & HIE861-Main-O & $38.8 \%$ \\
\hline \multirow[t]{3}{*}{6} & Pravastatin & LIG1-Side-O5 & ASP690-Side-OD1 & $91.10 \%$ \\
\hline & & LYS735-Side-NZ & LIG1-Side-O6 & $49.35 \%$ \\
\hline & & LYS735-Side-NZ & LIG1-Side-O5 & $44.70 \%$ \\
\hline 7 & Resveratrol & LIG788-Main-O & ASN658-Side-OD1 & $14.25 \%$ \\
\hline \multirow[t]{7}{*}{8} & Rosuvastatin & LYS735-Side-NZ & LIG788-Side-O5 & $74.90 \%$ \\
\hline & & LIG788-Main-O & GLU559-Side-OE2 & $60.50 \%$ \\
\hline & & LIG788-Main-O & GLU559-Side-OE1 & $56.60 \%$ \\
\hline & & LIG788-Side-O4 & ASP690-Side-OD1 & $39.15 \%$ \\
\hline & & LIG788-Side-O4 & ASP690-Side-OD2 & $36.90 \%$ \\
\hline & & LYS691-Side-NZ & LIG788-Main-O & $30.85 \%$ \\
\hline & & LIG788-Side-O1 & ASP690-Side-OD2 & $25.40 \%$ \\
\hline \multirow[t]{2}{*}{9} & Viniferin & LIG788-Side-O5 & ASP690-Side-OD1 & $63.85 \%$ \\
\hline & & LIG788-Side-O2 & GLY560-Main-O & $53.3 \%$ \\
\hline
\end{tabular}

\section{MMPBSA AND MMGBSA}

Some ligands were interacted with few of these residues carrying out hydrogen bonding and creating hydrophobic interaction. Rosuvastatin has sulphonyl functional group thus create hydrophilic properties and bind stronger compared to other statins. ${ }^{21}$ Ligands displayed susceptible affinity compared to statins, however, enacted better affinity than HMG. These ligands have slightly inhibitory activity to HMGCR although not as strong as statins are. At $310 \mathrm{~K}$, free binding energy tends to be lower as 
Table 3: Hydrogen bonds occupancy at $310 \mathrm{~K}$ during $20 \mathrm{~ns}$.

\begin{tabular}{|c|c|c|c|c|}
\hline No & Ligand & Donor & Acceptor & Occupancy (\%) \\
\hline 1 & Gnemonol M & LIG788-Side-O2 & GLU559-Side-OE1 & $42.20 \%$ \\
\hline \multirow[t]{2}{*}{2} & HMG & LYS735-Side-NZ & MAH788-Side-O1 & $48.85 \%$ \\
\hline & & MAH788-Side-O3 & GLU559-Side-OE1 & $40.80 \%$ \\
\hline \multirow[t]{4}{*}{3} & Gnetin C & LIG788-Side-O1 & GLU559-Side-OE1 & $67.95 \%$ \\
\hline & & LIG788-Side-O4 & LYS691-Main-O & $36.40 \%$ \\
\hline & & LIG788-Side-O3 & ASP690-Side-OD1 & $27.55 \%$ \\
\hline & & LIG788-Side-O1 & GLU559-Side-OE1 & $26.80 \%$ \\
\hline 4 & Gnemonoside B & UNK788-Side-O4 & HIE385-Side-OXT & $38.5 \%$ \\
\hline \multirow[t]{5}{*}{5} & Piceid & LIG788-Side-O6 & ASP690-Side-OD2 & $91.65 \%$ \\
\hline & & LIG788-Side-O1 & GLU559-Side-OE1 & $58.30 \%$ \\
\hline & & LIG788-Side-O2 & GLU559-Side-OE1 & $46.55 \%$ \\
\hline & & LIG788-Side-O1 & GLU559-Side-OE2 & $35.80 \%$ \\
\hline & & LIG788-Side-O2 & GLU559-Side-OE2 & $29.65 \%$ \\
\hline \multirow[t]{5}{*}{6} & Pravastatin & LIG1-Side-O4 & ASP690-Side-OD2 & $94.85 \%$ \\
\hline & & LIG1-Side-O5 & ASP690-Main-O & $77.80 \%$ \\
\hline & & ASN755-Side-ND2 & LIG1-Side-O2 & $50.50 \%$ \\
\hline & & LYS735-Side-NZ & LIG1-Side-O6 & $48.40 \%$ \\
\hline & & LIG1-Side-O2 & GLU559-Side-OE1 & $30.25 \%$ \\
\hline 7 & Resveratrol & LIG788-Main-O & ASP690-Side-OD2 & $24.40 \%$ \\
\hline \multirow[t]{5}{*}{8} & Rosuvastatin & LYS735-Side-NZ & LIG788-Side-O5 & $81.20 \%$ \\
\hline & & LIG788-Side-O4 & ASP690-Side-OD2 & $64.90 \%$ \\
\hline & & LIG788-Side-O1 & ASP690-Side-OD1 & $62.10 \%$ \\
\hline & & LIG788-Main-O & GLU559-Side-OE1 & $53.00 \%$ \\
\hline & & LIG788-Main-O & GLU559-Side-OE2 & $42.50 \%$ \\
\hline \multirow[t]{2}{*}{9} & Viniferin & LIG788-Side-O1 & GLU559-Side-OE1 & $32.60 \%$ \\
\hline & & LIG788-Side-O2 & CYS561-Main-O & $27.95 \%$ \\
\hline
\end{tabular}

Table 4: Recapitulation of MD affinity represented in $\mathrm{kcal} / \mathrm{mol}$.

\begin{tabular}{|c|c|c|c|c|c|}
\hline \multirow[t]{2}{*}{ No } & \multirow[t]{2}{*}{ Ligand } & \multicolumn{4}{|c|}{ Molecular Dynamic } \\
\hline & & Binding Affinity (kcal/mol) & Temperature (K) & MMPBSA (kcal/mol) & MMGBSA (kcal/mol) \\
\hline \multirow[t]{2}{*}{1} & Pravastatin & -10.08 & 300 & -27.2524 & -23.9170 \\
\hline & & & 310 & -29.6295 & -35.0508 \\
\hline \multirow[t]{2}{*}{2} & Rosuvastatin & -9.40 & 300 & -28.9507 & 29.8129 \\
\hline & & & 310 & -31.0991 & -30.8993 \\
\hline \multirow[t]{2}{*}{3} & Gnemonol M & -7.53 & 300 & -13.3906 & -11.0495 \\
\hline & & & 310 & -17.3838 & -12.6914 \\
\hline \multirow[t]{2}{*}{4} & HMG & -7.07 & 300 & -7.9018 & -10.6594 \\
\hline & & & 310 & -8.2101 & -14.1883 \\
\hline \multirow[t]{2}{*}{5} & Piceid & -7.07 & 300 & -19.0170 & -20.68882 \\
\hline & & & 310 & -15.7661 & -21.5739 \\
\hline \multirow[t]{2}{*}{6} & Resveratrol & -5.97 & 300 & -13.4773 & -14.7247 \\
\hline & & & 310 & -11.9587 & -15.7353 \\
\hline \multirow[t]{2}{*}{7} & Gnetin C & -7.33 & 300 & -13.2123 & -12.4022 \\
\hline & & & 310 & -19.3643 & -31.3852 \\
\hline \multirow[t]{2}{*}{8} & Viniferin & -7.39 & 300 & -14.3328 & -20.1497 \\
\hline & & & 310 & -17.0250 & -14.4980 \\
\hline \multirow[t]{2}{*}{9} & Gnemonoside B (aglycone) & -8.63 & 300 & -16.4978 & -13.4793 \\
\hline & & & 310 & -11.4466 & -11.7893 \\
\hline
\end{tabular}


shown in Gnetin C (Figure 5, 6). The increasing dynamic of the system develops more interactions, for instance, hydrogen bond contacts which bears stronger bond between ligands and residues.

\section{CONCLUSION}

On this simulation study, Gnetin C scored the best affinity.

\section{ACKNOWLEDGEMENT}

The authors are grateful to the University of Indonesia for providing necessary facilities and funding through PITTA 2017 to complete the paper.

\section{ABBREVIATIONS}

HMG-CoA: Hydroxymethylglutaryl-CoA; RMSD: Reductase; root mean square deviation; RMSF: Root mean square fluctuation; MMPBSA: hydrogen bonds analysis; MMGBSA: molecular mechanics Poisson Boltzmann surface area; molecular mechanics, generalized born surface area; CVD: Cardiovascular diseases.

\section{CONFLICT OF INTEREST}

The authors declare no conflict of interest.

\section{REFERENCES}

1. Djuwita R. Nutrient Intake and Plasma Low-Density Lipoprotein Cholesterol among Executive Group. DOI: http://dx.doi.org/10.21109/kesmas.v8i2.346.2007.

2. Taylor F, Huffman MD, Macedo AF, Moore THM, Burke M, Davey Smith G, et al. 2013. Statins for the primary prevention of cardiovascular disease. Cochrane database Syst Rev. 2011;1(1).CD004816.

3. Pan SY, Zhou SF, Gao SH, Yu ZL, Zhang SF, Tang MK, et al. 2013. New perspectives on how to discover drugs from herbal medicines: CAM'S outstanding contribution to modern therapeutics. Evidence-based Complement Altern Med. 2013

4. Ota H, Akishita M, Tani H, Tatefuji T, Ogawa S, lijima K, et al. Trans-resveratrol in Gnetum gnemon protects against oxidative-stress- induced endothelial senescence. J Nat Prod. 2013;76(7):1242-7.

5. Villanueva JA, Sokalska A, Cress AB, Ortega I, Bruner-Tran KL, Osteen KG, et al. Resveratrol Potentiates Effect of Simvastatin on Inhibition of Mevalonate Pathway in Human Endometrial Stromal Cells. J Clin Endocrinol Metab. 2013;98(3):E455-62.
6. Wang H, Yang YJ, Qian HY, Zhang Q, Xu H, Li JJ. Resveratrol in cardiovascular disease: What is known from current research? Vol. 17, Heart Failure Reviews. 2012:17(3)437-48

7. Hafidz KA. HMG-CoA Reductase Inhibitory Activity of Gnetum gnemon Seed Extract and Identification of Potential Inhibitors for LoweringCholesterol Level. J Young Pharm. 2017;9(4):559-65.

8. Karplus M, Mccammon JA. Molecular dynamics simulations of biomolecules. Nat Struct Biol. 2002;9(9):646-52.

9. Jakalian A, Jack DB, Bayly $\mathrm{Cl}$. Fast, efficient generation of high-quality atomic charges. AM1-BCC model: II. Parameterization and validation. J Comput Chem. 2002;23(16):1623-41.

10. Cho IJ, Ahn JY, Kim S, Choi MS, Ha TY. Resveratrol attenuates the expression of HMG-CoA reductase mRNA in hamsters. Biochem Biophys Res Commun. 2008;367(1):190-4.

11. Laskowski RA, Swindells MB. LigPlot+: Multiple ligand-protein interaction diagrams for drug discovery. J Chem Inf Model. 2011;51(10):2778-86.

12. Pearlman DA, Case DA, Caldwell JW, Ross WS, Cheatham TE, DeBolt S, et al. AMBER, a package of computer programs for applying molecular mechanics, normal mode analysis, molecular dynamics and free energy calculations to simulate the structural and energetic properties of molecules. Comput Phys Commun. 1995;91(1-3):1-41.

13. Meagher KL, Redman LT, Carlson HA. Development of Polyphosphate Parameters for Use with the AMBER Force Field. 2003;24(9):1016-25.

14. Humphrey W, Dalke A, Schulten K. VMD: Visual molecular dynamics. J Mol Graph. 1996;14(1):33-8.

15. Huey R, Morris GM. Using AutoDock with AutoDockTools: A Tutorial. The Scripps Research Institute Molecular Graphics Laboratory. 2008;8:54-6.

16. Morris GM, Lim-Wilby M. Molecular docking. Methods Mol Biol. 443:365-82.

17. Vendome J, Posy S, Jin X, Bahna F, Ahlsen G, Shapiro L, et al. Molecular design principles underlying $\beta$-strand swapping in the adhesive dimerization of cadherins. Nat Struct Mol Biol [Internet]. 2011;18(6):693-700. Available from: http://dx.doi. org/10.1038/nsmb.2051.

18. Desiraju GR. What is a weak hydrogen bond?. School of Chemistry University of Hyderabad. 2001.

19. Kästner J, Loeffler HH, Roberts SK, Martin-Fernandez ML, Winn MD. Ectodomain orientation, conformational plasticity and oligomerization of ErbB1 receptors investigated by molecular dynamics. J Struct Biol. 2009;167(2):117-28.

20. Istvan ES. Structural Mechanism for Statin Inhibition of HMG-CoA Reductase. Science (80- ) [Internet]. 2001;292(5519):1160-4. Available from: http://science. sciencemag.org/content/292/5519/1160.abstract.

21. White CM. A review of the pharmacologic and pharmacokinetic aspects of rosuvastatin. J Clin Pharmacol [Internet]. 2002;42(9):963-70. Available from: http://www.ncbi.nlm.nih.gov/pubmed/12211221.
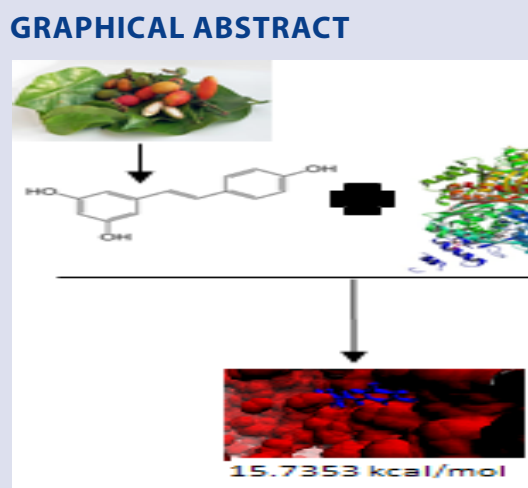

ABOUT AUTHORS

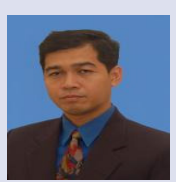

Dr. Arry Yanuar, M.Si.Farm., Apt. Achieved Bachelor degree from University of Indonesia in Faculty of Math and Science and continued the Apothecary program. He graduated from Magister program at University of Gadjah Mada majoring Pharmacy. He conducted research at National Institute of Health (NIH), Bethesda, USA (2000). He completed his doctoral program at Nara Institute of Science and Technology (NAIST). He is working at Faculty of Pharmacy since 1990 until now

Cite this article: Artha Y, Arrahman A, Azminah, Yanuar A. Molecular Dynamic Simulation of Hydroxymethylglutaryl-CoA Reductase Inhibitors from Gnetum Gnemon L. Seed Extract. Pharmacog J. 2018;10(4):793-7. 